\title{
On the Conversion between Content MathML and OpenMath
}

Clare M. So, Stephen M. Watt

Content MathML and OpenMath are two XML formats for semantic markup of mathematical expressions. Although efforts have been made to align their definitions, enough differences remain to make translation between them nontrivial.

In this chapter, we present a technical discussion of the differences between Content MathML and OpenMath, and two strategies for bidirectional translation between them. The first approach is to map between predefined Content MathML elements and standard OpenMath definitions, making any necessary adaptations for concepts that do not exactly correspond. For the second method, we describe a mapping using the extension mechanisms of each language, wherein all references to standard concepts are replaced by equivalent external references.

We have implemented these translation schemes (both approaches and both directions) as XSLT stylesheets. These implementations have served both to test our ideas and as components in an architecture for mathematical web services.

\section{Introduction}

Mathematical software packages, including computer algebra systems and theorem provers, define their own system-dependent syntax for commands and expressions. This is appropriate under the assumption that a person uses only a small number of such systems. The drawback of package-specific notation for mathematical content is that it makes data exchange between programs difficult and it limits a person's ability to take advantage of the strengths of unfamiliar systems.

OpenMath $[2,9,10]$ and Content MathML [4] were both devised to provide system-independent, non-proprietary formats for the semantics of mathematical expressions. They are complementary standards with different emphases. For example, OpenMath currently does not define how its expressions should be displayed, and can use MathML for that purpose. Conversely, Content MathML may be extended using OpenMath annotations to express semantic concepts that it does not natively provide. 
The relationship between OpenMath and MathML has been discussed elsewhere $[6,7,11]$.

There are two implications to the fact that the standards are complementary. First, since each standard may rely to some extent on the other, it is clear that the languages are needed simultaneously for the general management of mathematical knowledge (on the web or otherwise). Hence, translations between the languages are necessary. However, the co-dependence between the languages also implies that possibly they are different on a deeper level, that is, sufficiently mismatched so as to complicate such translations. Indeed, as we will describe, there are differences which prevent the simple translation of certain expressions from conserving semantics.

Our primary aim in this chapter is to give a detailed analysis of translation between the Content MathML and OpenMath formats. In doing so, we uncover certain discrepancies that may preclude the full interoperability of the languages, and which therefore should be taken into consideration in future versions of these standards.

We describe two strategies for translation between Content MathML and OpenMath, and we demonstrate how these mismatches may be addressed in both cases. The first strategy is to convert between pre-defined Content MathML elements and OpenMath's MathML Content Dictionaries, preserving the use of Content MathML's built-in semantics if possible. This translation also handles the primitives of both formats. The second strategy maps all semantics of the source into expressions using the extension mechanism of the target language. This open-ended strategy uses only the low-level expression forming primitives of OpenMath and Content MathML, and uses external definitions for all mathematical content. Both strategies match the basic elements of the standards.

To summarize, the principle contributions of this work are:

- A strategy for high-level conversion between OpenMath and Content MathML. We identify mismatches between Content MathML and OpenMath, including shortcomings of the OpenMath "MathML" Content Dictionary, intended to support Content MathML.

- A strategy for uniform conversion between OpenMath and MathML. The target language, either OpenMath or Content MathML, is used as a "carrier" syntax, and translations uniformly use the extension mechanisms.

The remainder of this chapter is organized as follows. Section 2 introduces the Content MathML and OpenMath standards. Section 3 presents the mapping between the semantics of Content MathML and OpenMath's MathML CD group. It also presents the discrepancies between Content 
MathML and OpenMath. Section 4 describes how we can always use external definitions in Content MathML when matching semantics are not available. This translation strategy helps us to overcome the cases in which OpenMath's semantics do not have Content MathML equivalent. Section 5 describes our implementation of a translator based on these ideas and Section 6 concludes the chapter.

\section{OpenMath and Content MathML}

The OpenMath and Content MathML standards both provide facilities to manage mathematical knowledge. Both formats serve to encode the semantics of mathematical expressions. Neither format is used to specify mathematical notation. Rather, it is expected that $\mathrm{T}_{\mathrm{EX}}$ or Presentation $M a t h M L$ can be used to represent expression notation, which is beyond the scope of this chapter. Before contrasting the two languages, we highlight their key characteristics individually.

2.1 OpenMath. OpenMath a system-independent XML [5] format to encode the semantics of mathematical expressions. It can be used as input and output for scientific computation as well as the format for mathematical expressions in documents. This standard includes a set of predefined elements describing some elementary concepts such as variables, floating point numbers and integers. No mathematical function is predefined. All semantics of the mathematical functions are defined in collections called Content Dictionaries (CDs). These CDs may be defined by a single application, by an agreement between two parties or by community consensus.

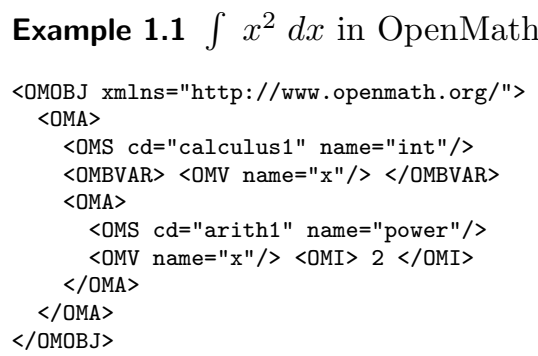

$\S$ Extending OpenMath. OpenMath is extensible by design. The semantics of mathematical functions are defined using Content Dictionaries, which may, in principle, be contributed by anyone. This removes the need to refer to semantics expressed in languages other than OpenMath. Existing, frequently-used mathematical functions and constants are pre-defined in official "standard" OpenMath CDs. 


\begin{tabular}{|l|l|}
\hline CD name & Description \\
\hline \hline alg1 & Some basic algebraic concepts \\
arith1 & Common arithmetic functions \\
bigfloat1 & Representation of floating point numbers \\
calculus1 & Calculus operations \\
complex1 & Operations and constructors of complex numbers \\
fns1 & Constructors and functions for functions \\
integer1 & Basic integer functions \\
linalg1 & Discrete and continuous 1-dimensional intervals \\
linalg2 & Operations on matrices \\
limit1 & Matrices and vectors in a row oriented fashion \\
list1 & Basic notion of the limits of unary functions \\
logic1 & List constructors \\
mathmltypes & Basic logic functions and constants \\
minmax1 & Types and constructs handled by MathML \\
multiset1 & Minimum and maximum of a set \\
nums1 & Basic multiset theory \\
piece1 & Common numerical constants and constructors \\
quant1 & Operators for piece-wise defined expressions \\
relation1 & Basic universal and existential quantifiers \\
setname1 & Common arithmetic operations \\
rounding1 & Common sets in mathematics \\
set1 & Basic rounding concepts \\
s_data1 & Functions and constructors for basic set theory \\
s_dist1 & Basic statistical functions used on sample data \\
transc1 & Basic statistical functions used on random variables \\
veccalc1 & Transcendental functions \\
altenc & Functions for vector calculus \\
\hline
\end{tabular}

Figure 2.1. OpenMath's MathML CD group.

The standard CDs and CD groups of OpenMath are organized so that related functions and constants are usually placed in the same CD. Related CDs then belong to a CD group. The OpenMath Society has already approved a certain number of CDs and CD groups. In the next section we examine one of the OpenMath Society's official CDs, the MathML CD group, shown in Figure 2.1. 
2.2 Content MathML. MathML [4] is a system-independent format to encode mathematical expressions for web documents. Different types of MathML markup are available to accommodate different applications of mathematics. Presentation markup provides a format for specifying the notation of a mathematical expression. Content markup provides a format for specifying the semantics and the structure of mathematical expressions without implying any actual notation being used. This type of markup allows applications to specify the notation, and process the semantics of the expression separately. Mixed markup associates the two types of markup by bundling together, at some level of resolution, the notation and the semantics of a mathematical expression. This type of markup allows a variety of applications to process the expression, using either the semantics or the notation.

Since we aim to convert between OpenMath and MathML, we are concerned only with the Content markup aspect of MathML. Currently, Content MathML consists of a base set of elements covering subjects in elementary mathematics. The subject areas covered by Content MathML to some extent include arithmetic, algebra, logic, relations, calculus, set theory, sequences and series, elementary classical functions, statistics and linear algebra.

Example 1.2 $\int x^{2} d x$ in Content MathML

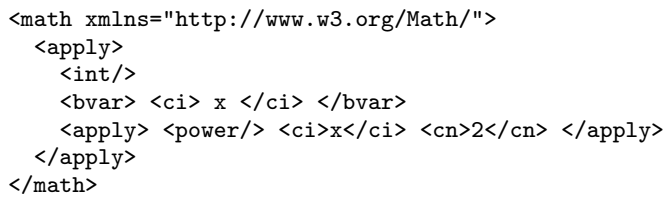

$\S$ Extending Content MathML. Content MathML's base set of elements cannot natively handle more than a fraction of the vast set of mathematical concepts. To overcome this problem, Content MathML provides an extension mechanism allowing one to redefine Content MathML elements or to introduce new mathematical semantics. The following examples illustrate the use of these extension mechanisms in Content MathML.

Example 1.3 Content MathML predefines <minus> as a unary or a binary arithmetic operator. If we would like to use this element as a n-ary operator, we have to override its semantics. This is accomplished by referring to an external URL. The definitionURL attribute gives a location defining the new semantics. The following Content MathML markup illustrates how one might encode the expression $1-2-3-4-5$ :

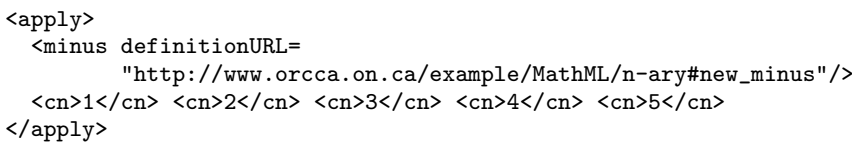



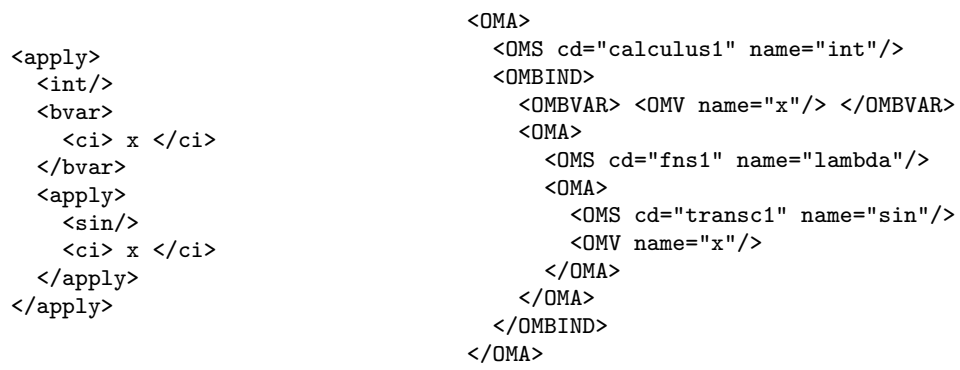

Figure 3.5. $\int \sin (x) d x$ in Content MathML (left) and OpenMath (right)

by the type attribute, which must be one of real, integer, rational, complex-cartesian, complex-polar or constant. If, as shown in Figure 3.4, an OpenMath number can be represented in one of the forms allowed by Content MathML <cn>, a special transformation can be applied. Otherwise, external semantics may be used or a composite Content MathML may be constructed (i.e. as an expression, rather than a single <cn>).

$\S$ Use of Lambda Bindings. In OpenMath, lambda bindings can be used to represent functions, as shown in Figure 3.5. For example, the calculus1 CD operators diff and int can take a function whose arguments are specified with lambda bindings. In Content MathML, however, bound variables are not specified as part of the function, but rather as arguments to the operators. So, in translating from OpenMath to Content MathML, if a function expression does not give an explicit lambda binding, then there is no way to determine the bound variables. If the argument of such functions in OpenMath is not a lambda binding, we may use heuristics to guess the bound variable when translating from OpenMath to Content MathML. In the reverse translation, we need to reconstruct a lambda binding from the function application with the bound variables.

$\S$ Miscellaneous Integer Functions. There is no direct Content MathML equivalent of OpenMath's trunc and round from the rounding1 CD, and abs from the arith1 CD. External references or special transformations constructing equivalent mathematical functions may be used.

We have seen that while the definitions of OpenMath and Content MathML allow many expressions to be translated precisely, there are several technical points on which the formats do not agree. Provided the application can be made to avoid these aspects, it is possible to have a natural translation between the corresponding concepts provided by the two standards. If a more robust solution is required, then another approach is needed, as described in the next section. 


\section{Implementation}

We have implemented a translator based on the strategies discussed in Sections 3 and 4 . The translator was implemented as a set of XSLT [8] stylesheets. The stylesheets are tested using xt [3], one of the implementations of XSLT. XSLT was chosen to implement the translator because it is designed specifically to transform XML expression trees. Another advantage is that many implementations of XSLT are free and widely available. The stylesheets are available at the website of our laboratory: http://www.orcca.on.ca/MathML.

There has been at least one other implementation of a Content MathML/ OpenMath translator [12]. This previous work discusses only the correspondence between the built-in semantics of the formats and is based in a REDUCE environment. In contrast, our open-ended conversion strategy does not restrict our attention to built-in semantics, and we have employed more widely available XML technology for our implementation.

It should be noted that our work is based on OpenMath version 1.0 and MathML 2.0. Since the development of our translator, both of the standards have evolved. At the time of writing, OpenMath 2.0 has been released and discussions for MathML 3.0 are underway. Although minor revisions will be made to the respective standards, our translation strategies can still be applied and the mismatches that we have identified here still exist.

\section{Conclusion}

OpenMath and Content MathML are two standards to encode the semantics of mathematical expressions. There are many similarities and differences between them.

We have described two translation strategies to convert between these two languages: The first strategy maps between the corresponding elements of the standards. It exposes a number of differences between OpenMath and Content MathML that must be dealt with. The second strategy uses the low-level structure of the formats to give precise embeddings of each within the other.

We have implemented these translation strategies using XSLT. The implementations have served two purposes: first as a proof of concept, to verify the validity of the approaches, and second, to fill a practical need, providing translator blocks in an architecture for mathematical web services [1]. 


\section{Bibliography}

[1] MONET (Mathematics on the Net), http://monet.nag.co.uk/cocoon/ monet/index.html.

[2] The OpenMath Society, http://www.openmath.org/.

[3] XT, http://www.jclark.com/xml/xt-old.html.

[4] R. Ausbrooks, S. Buswell, D. Carlisle, S. Dalmas, S. Devitt, A. Diaz, M. Froumentin, R. Hunter, P. Ion, M. Kohlhase, R. Miner, N. Poppelier, B. Smith, N. Soiffer, R. Sutor, and S. Watt, Mathematical Markup Language (MathML) Version 2.0 (second edition), W3C Recommendation 21 October 2003, World Wide Web Consortium (W3C), 2003, http://www.w3.org/TR/2003/REC-MathML2-20031021.

[5] Tim Bray, Jean Paoli, C.M. Sperberg-McQueen, Eve Maler, and cois Yergeau Fran Extensible Markup Language (XML) 1.0 (fourth edition) w3c Recommendation 16 August 2006, 2006, http://www.w3. org/TR/2006/REC-xml-20060816.

[6] David Carlisle, OpenMath, MathML, and XSL, ACM SIGSAM Bulletin 34 (2000), 6-11.

[7] David Carlisle, James Davenport, Mike Dewar, Namhyun Hur, and William Naylor, Conversion between MathML and OpenMath, The OpenMath Consortium (2001).

[8] James Clark, XSL Transformations (XSLT) Version 1.0, W3C Recommendation 16 November 1999, World Wide Web Consortium (W3C), 1999, http://www.w3.org/TR/xslt.

[9] Stéphane Dalmas, Marc Gaëtano, and Stephen Watt, An OpenMath 1.0 implementation, Proceedings of the 1997 International Symposium on Symbolic and Algebraic Computation (ISSAC), 1997, pp. 241-248.

[10] Mike Dewar, OpenMath: an overview, ACM SIGSAM Bulletin 34 (2000), $2-5$. 
[11] W.N. Naylor and S.M. Watt, On the relationship between OpenMath and MathML, Electronic Proc. Internet Accessible Mathematical Communication (IAMC 2001), http://icm.mcs.kent.edu/research/ iamc01proceedings.html.

[12] Luis Alvarez Sobreviela, A Reduce-based OpenMath $\leftrightarrow$ MathML translator, ACM SIGSAM Bulletin 34 (2000), 31-32. 